\title{
Direct Method Comparing Efficient and Nonefficient Payoffs in Differential Games ${ }^{1,2,3}$
}

\author{
J. P. Rincón-Zapatero ${ }^{4}$ ANd G. Martín-Herrán ${ }^{5}$ \\ Communicated by J. Shinar
}

\begin{abstract}
In this note, we present a method that allows us to decide when a Markov-perfect Nash equilibrium is not Pareto optimum, without the explicit knowledge of the respective solutions. For that purpose, we establish a sufficient condition in terms of an algebraic inequality where the gradient of the value functions of the cooperative and noncooperative games as well as the state and control variables are involved.
\end{abstract}

Key Words. Differential games, Markov-perfect Nash equilibrium, Pareto optimum, Hamilton-Jacobi-Bellman equations.

\section{Introduction}

The characterization and comparison of the optimal policies, when each player sets its policy in a noncooperative manner or when all players coordinate their actions and set their policy jointly, are issues which have received considerable attention in the differential game literature. The gains from policy coordination have been shown in different applied contexts, for example, environmental and economic problems. These results have been

\footnotetext{
${ }^{1}$ The first author acknowledges financial support from Junta de Castilla y León Project VA 108/01 and Dirección General de Investigación Project BFM2002-00425.

${ }^{2}$ The second author acknowledges financial support from JCYL Project VA051/03 and MCYT Project BEC2002-0236, cofinanced by FEDER funds.

${ }^{3}$ The authors thank the anonymous referees for helpful comments.

${ }^{4}$ Assistant Professor, Departamento de Economía Aplicada (Matemáticas), Facultad de Ciencias Económicas, Universidad de Valladolid, Valladolid, Spain.

${ }^{5}$ Assistant Professor, Departamento de Economía Aplicada (Matemáticas), Facultad de Ciencias Económicas, Universidad de Valladolid, Valladolid, Spain.
} 
established after both cooperative and noncooperative games have been solved completely.

In this note, we focus on the comparison of the total value obtained by the players when they follow either a cooperative or a noncooperative mode of play. More specifically, our objective is to see under which circumstances the total payoff under policy coordination is strictly greater than the sum of the individual payoffs in the noncooperative game. The question that we address is the following: Is there scope for cooperation in a particular differential game? In other words, is the cooperative surplus positive? The main contribution of the paper is that this question can be answered without the knowledge of explicit solutions to the Hamilton-Jacobi-Bellman (HJB) equations.

The question of the inefficiency of the noncooperative solution was studied first by Lancaster (Ref. 1) and later by Hoel (Ref. 2) in a differential game between workers and capitalists. In both papers, the open-loop equilibrium qualifies also as a Markov-perfect equilibrium that depends on time only, leading to a quite simple proof. Our aim in this paper is to provide a general result, applicable to a wide class of differential games and for Markov strategies. We establish a sufficient condition in terms of the fulfillment of an algebraic inequality involving the partial derivatives of the value functions of the cooperative and noncooperative games as well as the state and control variables. The main advantage of our approach is that it allows us to deduce if the Pareto strategies are better than the closed-loop Nash equilibria without knowing the respective solutions of the HJB equations, but only using the properties of the functions which satisfy these equations. The method is applied to analyze the inefficiency of feedback Nash equilibria in two different economic differential games.

\section{Description of the Game}

An $N$-person noncooperative differential game is determined by the following expressions:

$$
\begin{array}{r}
\max _{u^{i} \in U^{\prime}}\left\{J^{i}\left(t, x, u^{1}, \ldots, u^{N}\right)=\int_{t}^{T} L^{i}\left(s, y, u^{1}, \ldots, u^{N}\right) d s+S^{i}(T, x(T))\right\}, \\
i=1, \ldots, N,
\end{array}
$$

s. t. $\dot{y}=f\left(s, y, u^{1}, \ldots, u^{N}\right)$,

$$
y(t)=x, \quad y \in X \subseteq \mathbb{R}^{n}, \quad U^{i} \subseteq \mathbb{R}^{m_{i}}, \quad t_{0} \leq t \leq T, \quad t_{0} \geq 0,
$$


where $y=\left(y^{1}, \ldots, y^{n}\right)^{\top}$ and $u^{i}=\left(u^{i_{1}}, \ldots, u^{i_{m_{i}}}\right)^{\top}$ are the state and control variables for the $i$ th player, respectively, and the superscript $T$ denotes the transposition operation. The time interval $\left[t_{0}, T\right]$ is assumed to be fixed ( $T=+\infty$ is allowed) and the functions $L^{i}, S^{i}, f$ are continuous in their arguments and continuously differentiable. The admissible strategies for the $i$ th player are of Markov type, that is, functions $\phi^{i}:\left[t_{0}, T\right] \times X \rightarrow U^{i}$ continuous almost everywhere and such that system (2)-(3) admits a unique absolutely continuous solution.

We refer the reader to Refs. 3-4 for the definitions of Markov-perfect Nash equilibrium and Pareto optimum, as well as for their characterizations by means of the HJB equations.

It is well known that a sufficiency condition for Pareto optimality can be stated in the following terms (Ref. 3). A profile of strategies $\tilde{\phi}=\left(\tilde{\phi}^{1}, \ldots, \tilde{\phi}^{N}\right)$ is Pareto optimal if there exist $\alpha_{i} \in(0,1), i=1, \ldots, N$, $\sum_{i=1}^{N} \alpha_{i}=1$, such that, for all $N$-tuple of strategies $\phi=\left(\phi^{1}, \ldots, \phi^{N}\right)$,

$$
\begin{aligned}
& \sum_{i=1}^{N} \alpha_{i} J^{i}\left(t, x, \tilde{\phi}^{1}, \ldots, \tilde{\phi}^{N}\right) \\
& \geq \sum_{i=1}^{N} \alpha_{i} J^{i}\left(t, x, \phi^{1}, \ldots, \phi^{N}\right), \quad \text { for all } t, x \text { admissible. }
\end{aligned}
$$

Hence, the solution to the cooperative game can be obtained by means of the solution to the following optimal control problem:

$$
\begin{array}{ll}
\max _{u \in U} & \sum_{i=1}^{N} \alpha_{i} J^{i}\left(t, x, u^{1}, \ldots, u^{N}\right), \\
\text { s.t. } & \dot{y}=f\left(s, y, u^{1}, \ldots, u^{N}\right), \\
& y(t)=x, \quad x \in X \subseteq \mathbb{R}^{n}, \quad U^{i} \subseteq \mathbb{R}^{m_{i}},
\end{array}
$$

where

$$
U=U^{1} \times \cdots \times U^{N}, \quad 0 \leq \alpha_{i} \leq 1, \quad \sum_{i=1}^{N} \alpha_{i}=1 .
$$

Let $V^{i}$, with $i=1, \ldots, N$, and let $W^{\alpha}$, with $\alpha=\left(\alpha_{1}, \ldots, \alpha_{N}\right)$, be continuously differentiable functions, defined in the set $\left[t_{0}, T\right] \times X$, which satisfy the HJB equations and final conditions associated with the noncooperative and cooperative games, respectively.

\section{Inefficiency of Markov-Perfect Nash Equilibria}

In this section, we center on the comparison of the global value that the players obtain when they coordinate their strategies and when they do 
not. More specifically, we study whether the global value associated with a cooperative mode of play is strictly greater than that obtained when the players play in a decentralized way, that is, whether $\sum_{i=1}^{N} \alpha_{i} J^{i}\left(t, x, u^{\alpha}\right)$ is strictly greater than $\sum_{i=1}^{N} \alpha_{i} J^{i}\left(t, x, u^{*}\right)$, where $u^{\alpha}$ is a feedback Pareto optimum and $u^{*}$ denotes a Markov-perfect Nash equilibrium of the noncooperative game. This is an important issue; indeed, if this holds, noncooperation would lead to an inefficient result that would be improved if players cooperate. The next theorem allows to answer this question for the Markov-perfect Nash equilibria, without the explicit knowledge of the solutions to the HJB equations.

In the next result, $\langle\cdot, \cdot\rangle$ denotes the usual scalar product in $\mathbb{R}^{n}$ and $\nabla$ is the gradient operator.

Theorem 3.1. Let $V^{i}, i=1, \ldots, N$, and let $W^{\alpha}$ be continuously differentiable functions for all $i$ and for all $(t, x) \in\left[t_{0}, T\right] \times X$. Assume that, for $t_{0} \leq t \leq T$ and $x \in X$, there exists a vector of strategies $v=\left(v^{1}, \ldots, v^{N}\right)$ such that (2) admits a unique absolutely continuous solution $\check{X}$ and

$$
\left\langle\nabla\left(W^{\alpha}-\sum_{i=1}^{N} \alpha_{i} V^{i}\right)(s, y),(1, f(s, y, v))\right\rangle \leq 0, \quad \text { for all } y \in X,
$$

with strict inequality for some subset $E_{t} \subseteq[t, T]$ of positive Lebesgue measure. If $W^{\alpha}(T, \check{x}(T)) \geq \sum_{I=1}^{N} V^{i}(T, \check{X}(T))$, then

$$
\sum_{i=1}^{N} \alpha_{i} J^{i}\left(t, x, u^{\alpha}\right)>\sum_{i=1}^{N} \alpha_{i} J^{i}\left(t, x, u^{*}\right)
$$

For all vectors $v=\left(v^{1}, \ldots, v^{N}\right)$, if (7) is an equality for all $y$ and for almost every $s \in[t, T]$, then the Markov-perfect Nash equilibrium is a Pareto optimum.

Proof. Let $t \geq t_{0}$ and $x \in X$, and let $\check{x}$ be the trajectory associated with $v$, that is,

$$
\check{x}(s)=\check{x}(t)+\int_{t}^{s} f(r, \check{x}(r), v(r, \check{x}(r))) d r, \quad \check{x}(t)=x, s \geq t .
$$

This function is absolutely continuous and therefore differentiable in almost every point of $[t, T]$. Let us define the function

$$
g(s)=W^{\alpha}(s, \check{x}(s))-\sum_{i=1}^{N} \alpha_{i} V^{i}(s, \check{x}(s)), \quad t \leq s \leq T .
$$


Let $A$ be the subset of $[t, T]$ such that either $\check{x}$ is not differentiable or condition (7) is not satisfied. By hypothesis, this is a set of zero Lebesgue measure. In $[t, T]-A, g^{\prime}(\cdot) \leq 0$ is satisfied, since the application of the chain rule yields that the derivative of function $g$ is the left-hand side expression of inequality (7). On the other hand, since $W^{\alpha}-\sum_{i=1}^{N} V^{i}$ is continuously differentiable and is locally Lipschitz, and since $\check{x}$ is absolutely continuous in the compact interval $[t, T]$, so is the function $s \mapsto h(s)=(s, \check{x}(s))$; therefore, the composition $\left(W^{\alpha}-\sum_{i=1}^{N} \alpha_{i} V^{i}\right) \circ h$, that is, the function $g$, is absolutely continuous (Ref. 5). But a function is absolutely continuous if and only if it can be expressed as

$$
g(s)=g(t)+\int_{t}^{s} g^{\prime}(r) d r
$$

for all $s \in[t, T]$. Applying the properties of the Lebesgue integral and taking into account the above representation of function $g$, one can deduce that, since $g^{\prime}$ is defined and is not positive in almost all points of $[t, T]$, the function $g$ is monotonous nonincreasing in such interval. In fact, $g^{\prime}<0$ in $E_{t}$ and then $g(t)>g(T)$, since $g$ is strictly decreasing in a subset of positive Lebesgue measure.

To conclude, let us observe that

$$
\begin{aligned}
g(t) & =W^{\alpha}(t, x)-\sum_{i=1}^{N} \alpha_{i} V^{i}(t, x) \\
& =\sum_{i=1}^{N} \alpha_{i}\left(J^{i}\left(t, x, u^{\alpha}\right)-J^{i}\left(t, x, u^{*}\right)\right), \\
g(T) & =W^{\alpha}(T, \check{x}(T))-\sum_{i=1}^{N} \alpha_{i} V^{i}(T, \check{x}(T)) \\
& =\sum_{i=1}^{N} \alpha_{i}\left[S^{i}(T, \check{x}(T))-S^{i}(T, \check{x}(T))\right] \geq 0 .
\end{aligned}
$$

Given that $g(t)>g(T)$, inequality (8) is deduced.

For all vectors $v=\left(v^{1}, \ldots, v^{N}\right)$, when (7) is an equality for all $x$ and for almost every $s \in[t, T]$, along the previous line it can be proved that $g^{\prime}=0$ and then

$$
\sum_{i=1}^{N} \alpha_{i} J^{i}\left(t, x, u^{\alpha}\right)=\sum_{i=1}^{N} \alpha_{i} J^{i}\left(t, x, u^{*}\right)
$$

Therefore, the Markov-perfect Nash equilibrium is a Pareto optimum. 


\section{Remark 3.1.}

(i) Since in (7) only the partial derivatives of $W^{\alpha}, V^{i}$, and the function $f$ are involved, the relationship between the cooperative and noncooperative games can be studied without the explicit knowledge of the solutions to the HJB equations. It is only necessary to know the behavior of the partial derivatives with respect to $x$, since the partial derivatives with respect to the time variable are related to the previous ones by means of these equations.

(ii) Notice that vector $v=\left(v^{1}, \ldots, v^{N}\right)$ is not restricted to the set of admissible controls, whenever the state equation admits a unique absolutely continuous solution.

(iii) The result is also applicable if $T=+\infty$ and

$\liminf _{T \rightarrow \infty}\left[W^{\alpha}(T, \check{x}(T))-\sum_{i=1}^{N} \alpha_{i} V^{i}(T, \check{x}(T))\right] \geq 0$.

In this case,

$\liminf _{t \rightarrow \infty} g(t) \geq 0$

and the same proof applies.

(iv) The hypotheses about the differentiability of $V^{i}$ and $W^{\alpha}$ can be weakened to the requirement of local Lipschitz continuity.

\section{Application to Economic Games}

In this section, we apply the result of Theorem 3.1 to two economic differential games: a slight extension of the well-known Lancaster model and a transboundary pollution problem. These models show that our theoretical result can be applied to different types of games. The first game presents bang-bang solutions, while the second game corresponds to a linearquadratic specification.

Example 4.1. As in the Lancaster model (Ref. 1), we assume an economy which produces a unique output, with only two different economic agents, workers and firms. The production $Y$ depends on two factors, the capital $K$ and labor $L$, by means of a first-degree homogeneous CobbDouglas function,

$$
Y=K^{\alpha} L^{\beta}, \quad \text { where } \alpha, \beta>0 \text { and } \alpha+\beta=1 .
$$

The workers (first player) decide the portion of the output which they consume $s Y$, where $0 \leq s_{1} \leq s \leq s_{2}<1$. On the other hand, the firms (second 
player) control which portion $\theta Y$ of the product they invest and also decide the labor supply $L$. We consider that

$$
0 \leq \theta \leq 1 \text { and } 0 \leq L \leq \mu K,
$$

where the positive constant $\mu$ depends on the technology of the productive process. The objective of both players is to maximize their own consumption over a fixed period of time $[0, T]$, taking into account the time evolution of the capital $K$. The game is specified completely by

$$
\begin{array}{ll}
\max _{s} & \left\{J^{1}(s, \theta, L)=\int_{0}^{T} s K^{\alpha} L^{\beta} d t\right\}, \\
\max _{\theta, L} & \left\{J^{2}(s, \theta, L)=\int_{0}^{T}(1-s)(1-\theta) K^{\alpha} L^{\beta} d t\right\}, \\
\text { s.t. } \quad & \dot{K}=(1-s) \theta K^{\alpha} L^{\beta}, \quad K(0)=K_{0}>0, \\
& 0 \leq s_{1} \leq s \leq s_{2}<1, \quad 0 \leq \theta \leq 1, \quad 0 \leq L \leq \mu K, \\
& \alpha>0, \quad \beta>0, \quad \alpha+\beta=1 .
\end{array}
$$

It can be proved that, in the optimal solution,

$$
L=\mu K \text {. }
$$

The HJB equation for the first player reads

$$
V_{t}^{1}+\mu^{\beta} K\left(s^{*}+V_{K}^{1}\left(1-s^{*}\right) \theta^{*}\right)=0,
$$

where

$$
s^{*}= \begin{cases}s_{1}, & \text { if } \theta^{*} V_{K}^{1}>1, \\ d_{1}, & \text { if } \theta^{*} V_{K}^{1}=1, \\ s_{2}, & \text { if } \theta^{*} V_{K}^{1}<1,\end{cases}
$$

$d_{1} \in\left[s_{1}, s_{2}\right]$ and $V^{1}$ denotes the value function of this player. The equation for the second player is

$$
V_{t}^{2}+\mu^{\beta} K\left[\left(1-s^{*}\right)\left(1-\theta^{*}+V_{K}^{2}\left(1-s^{*}\right) \theta^{*}\right)\right]=0,
$$

where

$$
\theta^{*}=\left\{\begin{array}{cc}
0, & \text { if } V_{K}^{2}<1, \\
d_{2}, & \text { if } V_{K}^{2}=1, \\
1, & \text { if } V_{K}^{2}>1,
\end{array}\right.
$$


$d_{2} \in[0,1]$, and $V^{2}$ denotes the value function of the second player. Both equations have to satisfy the final condition

$$
V^{i}(T, K)=0, \quad i=1,2 .
$$

From the optimality conditions, one can show the following: First, singular controls $s^{*}=d_{1}$ and $\theta^{*}=d_{2}$ can be excluded from the optimal solution; second, following a similar reasoning as in Lancaster (Ref. 1), one can assert that, close to the final time, $\left[t^{*}, T\right]$, the optimal strategy is

$$
\left(s^{*}(t), \theta^{*}(t)\right)=\left(s_{2}, 0\right) .
$$

We get three pairs of optimal strategies:

$$
\begin{array}{ll}
\left(s_{1}, 1\right), & \text { if } V_{K}^{1}>1, V_{K}^{2}>1 ; \\
\left(s_{2}, 0\right), & \text { if } V_{K}^{2}<1, \text { no matter what } V_{K}^{1} \text { is; } \\
\left(s_{2}, 1\right), & \text { if } V_{K}^{1}<1, V_{K}^{2}>1,
\end{array}
$$

knowing that this second case either applies always for all the time horizon or at least during a final subinterval.

When the same weight $\left(\alpha_{i}=1 / 2, i=1,2\right)$ is assigned to each player, the $\mathrm{HJB}$ equation for the cooperative game is given by

$$
W_{t}+\mu^{\beta} K\left(1 / 2 s^{p}+(1 / 2)\left(1-s^{p}\right)\left(1-\theta^{p}\right)+W_{K}\left(1-s^{p}\right) \theta^{p}\right)=0,
$$

where

$$
s^{p}= \begin{cases}s_{1}, & \text { if } W_{K} \theta^{p}>(1 / 2) \theta^{p}, \\ d_{1}^{p}, & \text { if } W_{K} \theta^{p}=(1 / 2) \theta^{p}, \\ s_{2}, & \text { if } W_{K} \theta^{p}<(1 / 2) \theta^{p},\end{cases}
$$

and

$$
\theta^{p}= \begin{cases}0, & \text { if } W_{K}<1 / 2, \\ d_{2}^{p}, & \text { if } W_{K}=1 / 2, \\ 1, & \text { if } W_{K}>1 / 2,\end{cases}
$$

$d_{1}^{p} \in\left[s_{1}, s_{2}\right], d_{2}^{p} \in[0,1]$, and $W$ denotes the value function for the cooperative game.

As in the noncooperative game, the singular control $\theta^{p}=d_{2}^{p}$, can be excluded. Only two possibilities are feasible:

$$
\left(s_{1}, 1\right), \quad \text { if } W_{K}>1 / 2 \text {, }
$$

and

$$
\left(d_{1}^{p}, 0\right), \quad \text { if } W_{K}<1 / 2 .
$$


When $\left(s_{2}, 0\right)$ is played in the noncooperative game, while $\left(s_{1}, 1\right)$ is played in the cooperative game, inequality (7) can be written as

$$
\left(\frac{1}{2}-W_{K}\left(1-s_{1}\right)+\left(W_{K}-\frac{1}{2} V_{K}^{1}-\frac{1}{2} V_{K}^{2}\right)(1-s) \theta \leq 0 .\right.
$$

Choosing any value of $s$ and $\theta=0$ in $[0, T]$, the left-hand side of the last inequality becomes $\left(1 / 2-W_{K}\right)\left(1-s_{1}\right)$, which is strictly lower than zero, since $W_{K}>1 / 2$ and $s_{1}<1$. Then, satisfaction of inequality (8) is guaranteed.

On the other hand, when $\left(s_{2}, 0\right)$ is still played in the noncooperative game, but $\left(d_{1}^{p}, 0\right)$ is played in the cooperative game, inequality (7) reads

$$
\left(W_{K}-\frac{1}{2} V_{K}^{1}-\frac{1}{2} V_{K}^{2}\right)(1-s) \theta \leq 0 .
$$

Again choosing any value of $s$ and $\theta=0$ in $[0, T]$ the left-hand side becomes identically null.

To sum up, in both cases, an efficient solution leading to a global consumption greater or equal than that associated with the Nash equilibrium can be guaranteed.

Example 4.2. We consider now the following $N$-country transboundary pollution differential game borrowed from Ref. 6:

$$
\begin{array}{ll}
\max _{Y_{i}} & \left\{J^{i}\left(Y_{i}, Y_{j}\right)=\int_{0}^{\infty} e^{-r t}\left[B\left(Y_{i}\right)-D(S)\right] d t\right\}, \quad i=1, \ldots, N, \\
\text { s.t. } & \dot{S}=(\alpha / N) \sum_{j=1}^{N} Y_{j}-\delta S, \quad S(0)=S_{0},
\end{array}
$$

where

$$
B\left(Y_{i}\right)=\beta Y_{i}-(1 / 2) Y_{i}^{2}
$$

is the net social benefits of the production $Y_{i}$ of country $i$ and

$$
D(S)=(1 / 2) \gamma S^{2}
$$

is the social damage caused by the stock of pollution $S$. The constants $\alpha$ and $\delta$ denote the emission-output ratio and the depreciation rate of the pollution concentration.

Let $V^{i}$ denote the value function of the $i$ th player. The HJB equation for this player reads

$$
-r V^{i}+\beta Y_{i}^{*}-(1 / 2)\left(Y_{i}^{*}\right)^{2}-(1 / 2) \gamma S^{2}+V_{S}^{i}\left(\alpha / N \sum_{j=1}^{N} Y_{j}^{*}-\delta S\right)=0,
$$

where

$$
Y_{i}^{*}=\beta+\alpha / N V_{S}^{i}
$$


Let $W$ denote the value function for the cooperative game when the same weight $\alpha_{i}=1 / N, i=1, \ldots, N$, is assigned to each player. For this game, the HJB equation is

$$
-r W+\beta Y^{p}-(1 / 2)\left(Y^{p}\right)^{2}-(1 / 2) \gamma S^{2}+W_{S}\left(\alpha Y^{p}-\delta S\right)=0,
$$

where

$$
Y^{p}=Y_{i}^{p}=\beta+\alpha W_{S}, \quad \text { for all } i=1, \ldots, N .
$$

It is straightforward to show that the conditions in Remark 3.1 (iii) apply.

Since the game is symmetric, we focus on symmetric solutions. Denoting $V=V^{i}, V_{S}=V_{S}^{i}$, and $Y=Y_{i}, i=1, \ldots, N$, inequality (7) can be written as

$$
-r W+r V+\left(W_{S}-V_{S}\right)(\alpha Y-\delta S) \leq 0 .
$$

Using the HJB equations for the cooperative and noncooperative cases, the last inequality reads

$$
\begin{aligned}
& -\alpha \beta\left(W_{S}-V_{S}\right)-\left(\alpha^{2} / 2\right)\left(W_{S}\right)^{2} \\
& +\left[(2 N-1) /\left(2 N^{2}\right)\right] \alpha^{2}\left(V_{S}\right)^{2}+\left(W_{S}-V_{S}\right) \alpha Y \leq 0 .
\end{aligned}
$$

If $W_{S}=V_{S}$, inequality (13) reads

$$
-\alpha^{2}\left[(N-1)^{2} /\left(2 N^{2}\right)\right]\left(V_{S}\right)^{2}<0 .
$$

If $W_{S} \neq V_{S}$, the left-hand side of inequality (13) can be bounded by

$$
\left(W_{S}-V_{S}\right)\left[-\alpha \beta-\left(\alpha^{2} / 2\right)\left(W_{S}+V_{S}\right)+\alpha Y\right] .
$$

Therefore, to apply Theorem 3.1, it is sufficient to show that

$$
\left(W_{S}-V_{S}\right)\left[-\alpha \beta-\left(\alpha^{2} / 2\right)\left(W_{S}+V_{S}\right)+\alpha Y\right] \leq 0 .
$$

If $W_{S}>V_{S}$, the last inequality is fulfilled if and only if

$$
Y \leq \beta+(\alpha / 2)\left(W_{S}+V_{S}\right)
$$

If $W_{S}<V_{S}$, the above inequality is satisfied if

$$
Y \geq \beta+(\alpha / 2)\left(W_{S}+V_{S}\right)
$$

Therefore, in any case it can be proved that the cooperative solution gives a larger welfare than the global welfare derived following a Nash equilibrium strategy. 


\section{References}

1. Lancaster, K., The Dynamic Inefficiency of Capitalism, Journal of Political Economy, Vol. 87, pp. 1092-1109, 1973.

2. Hoel, M., Distribution and Growth as a Differential Game between Workers and Capitalists, International Economic Review, Vol. 19, pp. 335-350, 1978.

3. Leitmann, G., Cooperative and Noncooperative Many-Players Differential Games, Springer Verlag, New York, NY, 1974.

4. Basar, T., and Olsder, G. J., Dynamic Noncooperative Game Theory, SIAM, Philadelphia, Pennsylvania, 1999.

5. Halmos, P. R., Measure Theory, Springer Verlag, New York, NY, 1974.

6. Van der Ploeg, F., and De Zeeuw, A. J., International Aspects of Pollution Control, Environmental and Resource Economics, Vol. 2, pp. 117-139, 1992. 